\title{
Mines et environnement dans les Amériques : les paradoxes de l'exploitation minière
}

Mining and Environment in the Americas: paradoxes of mining

Minas y medio ambiente en las Américas: las paradojas de la explotación minera

\section{Michel Deshaies}

\section{OpenEdition}

\section{Journals}

\section{Édition électronique}

URL : https://journals.openedition.org/ideas/1639

DOI : $10.4000 /$ ideas. 1639

ISSN : $1950-570$

\section{Éditeur}

Institut des Amériques

\section{Référence électronique}

Michel Deshaies, «Mines et environnement dans les Amériques : les paradoxes de l'exploitation minière », IdeAs [En ligne], 8| 2016, mis en ligne le 14 décembre 2016, consulté le 18 octobre 2022. URL : http://journals.openedition.org/ideas/1639; DOI : https://doi.org/10.4000/ideas.1639

Ce document a été généré automatiquement le 18 octobre 2022.

\section{(i) $\odot$

Creative Commons - Attribution - Pas d'Utilisation Commerciale - Pas de Modification 4.0 International - CC BY-NC-ND 4.0

https://creativecommons.org/licenses/by-nc-nd/4.0/ 


\title{
Mines et environnement dans les Amériques : les paradoxes de l'exploitation minière
}

\author{
Mining and Environment in the Americas: paradoxes of mining
}

Minas y medio ambiente en las Américas: las paradojas de la explotación minera

\author{
Michel Deshaies
}

\section{Introduction}

1 La richesse minière légendaire des Amériques reste aujourd'hui encore une réalité, puisque plus de la moitié de la valeur de la production mondiale de minerais métallifères vient du Nouveau Monde, en particulier l'or, l'argent et le cuivre. Plus de quatre siècles d'exploitation prédatrice ont laissé de nombreuses traces dans les paysages et l'environnement qui, jusqu'aux années soixante-dix, n'étaient pas pris en considération. Bien que les sociétés minières soient désormais soumises à des réglementations environnementales plus sévères que par le passé, l'impact de leur activité est aussi beaucoup plus important en raison de la forte croissance de l'extraction depuis les années soixante-dix. Celle-ci a été rendue possible par le développement de l'exploitation de nouveaux gisements à ciel ouvert dont l'empreinte sur les paysages atteint des dimensions extraordinaires. Le creusement d'immenses découvertes, les énormes volumes de déblais déplacés et les traitements chimiques employés entraînent, une destruction des paysages et du cadre de vie des communautés locales d'habitants.

Cependant, l'impact grandissant de cette exploitation minière à grande échelle soulève une opposition croissante de la part des communautés d'habitants concernées qui craignent en premier lieu une altération et des pollutions irrémédiables des ressources en eau. En Amérique latine en particulier, les conflits se sont multipliés à partir des années quatre-vingt-dix. Ces conflits où l'environnement est en quelque sorte " un champ de bataille entre les intérêts divergents » (Blaikie P., 1995) des firmes et des 
populations locales soutenues par les mouvements écologistes, exercent une pression croissante sur les projets miniers dont certains doivent être abandonnés. Les compagnies minières se retrouvent ainsi dans une situation paradoxale où leurs pratiques sont remises en cause, alors qu'en raison des réglementations environnementales elles sont soumises à des contraintes particulièrement sévères. Aussi sont-elles amenées à mettre en place des actions destinées à démontrer que leur activité est environnementalement et socialement responsable; ce qui ne garantit d'ailleurs pas le succès. Pourtant, pour que les Amériques puissent rester le principal fournisseur mondial de minerais métallifères, il faut que de nouveaux projets miniers puissent se développer afin de prendre le relais des mines en cours d'épuisement. Cet article examine la stratégie que doivent adopter les compagnies minières sur un continent marqué par l'héritage séculaire d'une exploitation prédatrice qui continue encore aujourd'hui à servir de repoussoir.

\section{La recherche de l'Eldorado et les héritages de l'exploitation prédatrice}

\section{Le rôle essentiel des ruées vers l'or dans le peuplement des Amériques}

3 L'une des motivations principales qui a poussé les Conquistadores à conquérir l'empire aztèque, puis l'empire inca au XVI ${ }^{e}$ siècle, les Européens à s'aventurer au XIX ${ }^{e}$ siècle dans les vastes déserts de l'Ouest américain, ou dans les solitudes glacées de l'Alaska ou du Yukon est la recherche de minerais précieux. Même si cette quête du mythique Eldorado se révéla la plupart du temps décevante, elle n'en déboucha pas moins sur de nombreuses et parfois fabuleuses découvertes telles que le gisement du Cerro Rico (la montagne riche) à Potosi où, dès 1545 on commença à exploiter la veine d'argent natif la plus pure du monde (Coulson M., 2012). Au milieu du XVII e siècle, le gisement de Potosi fournissait plus de la moitié de la production totale d'argent du Nouveau Monde et la ville la plus haute du monde (4090 m d'altitude) était devenue un vaste complexe industriel comptant près de 200000 habitants !

4 Le peuplement des montagnes de l'Ouest américain, dans la deuxième moitié du XIX siècle, est également essentiellement dû aux ruées vers l'or (Gold Rush) qui ont entraîné la venue de milliers d'aventuriers en Californie (1849), au Colorado (1858), au Montana (1864), en Colombie britannique, puis en Alaska (1902). Après la découverte de l'or alluvionnaire de Californie, épuisé en quelques années, les prospecteurs s'aventurèrent dans toutes les montagnes de l'Ouest américain, à la recherche de la fabuleuse veine mère, c'est-à-dire des filons renfermant une concentration assez forte d'or ou d'argent pour pouvoir être extrait avec les techniques de l'époque.

5 Mais les richesses minières exploitées étaient le plus souvent éphémères, les moyens techniques de l'époque ne permettant pas de récupérer le métal quand sa concentration était trop faible. Aussi, après l'épuisement des placers aurifères ou des veines superficielles, les villes minières déclinèrent aussi vite qu'elles avaient été créées. Beaucoup devinrent des villes fantômes (Ghost Town) totalement abandonnées, ou quand elles ne disparurent pas complètement, la population diminua énormément. 


\section{Le développement de la grande exploitation minière industrielle}

6 Rares en définitive sont les gisements qui, comme la mine de Homestake, dans les Black Hills, ont eu une longévité importante. En effet, découverts en 1876, l'or et l'argent y ont été exploités jusqu'à la fin du XXe siècle; si bien que la mine qui, en un siècle, a fourni près de 1000 tonnes d'or, s'est avérée être l'une des plus rentables au monde. Mais cette longévité est surtout due au fait que dès les années 1880, les chercheurs d'or aventuriers ont commencé à céder la place à des industriels recourant à des études géologiques et employant de nouvelles techniques permettant d'extraire l'or ou l'argent dans des minerais à faible, et même à très faible teneur. C'est ainsi que dès 1887 , est mis au point le procédé de la cyanuration qui permet de récupérer jusqu'à plus de $97 \%$ de l'or contenu dans le minerai, au lieu de 60 à $75 \%$ au mieux avec le procédé traditionnel de l'amalgamation au mercure. Mais pour être rentable sur des minerais à faible teneur, ce procédé exige d'être en mesure de traiter de grands volumes de minerais; ce que seules des sociétés pouvant mobiliser d'importants capitaux pouvaient réaliser. Aussi, dès le début du $\mathrm{XX}^{\mathrm{e}}$ siècle, l'essentiel de la production de minerais précieux des Amériques était réalisée par les grandes compagnies comme la Newmont Mining Corporation ou la société de Homestake (Coulson M., 2012).

7 Tout au long du XXe siècle, l'importance de ces compagnies s'est accrue et le rôle du prospecteur aventurier est devenu marginal. Employant des moyens mécaniques de plus en plus puissants, elles ont créé des paysages miniers originaux, marqués par la démesure, avec la création de gigantesques exploitations à ciel ouvert. L'exploitation minière reste toutefois majoritairement souterraine jusqu'aux années soixante-dix, même s'il existe déjà de grandes exploitations à ciel ouvert de cuivre, comme Bingham Canyon dans l'Utah, ou Chuquicamata au Chili.

\section{Les nombreux héritages environnementaux de l'exploitation minière}

8 Partout dans le monde, l'exploitation minière a revêtu longtemps un caractère prédateur et dévastateur pour l'environnement. Cette exploitation s'est exprimée avec une intensité particulière dans le Nouveau Monde où la législation a même encouragé l'esprit pionnier et l'extraction sans retenue des ressources naturelles. Cherchant à faire fortune ou à assurer une rentabilité immédiate et maximale, les petits prospecteurs comme les compagnies minières ont longtemps utilisé les moyens les plus polluants dévastateurs pour l'environnement et dès que le filon était épuisé, l'exploitation était brutalement fermée, sans se soucier des problèmes environnementaux que pouvaient générer les héritages miniers. C'est ainsi que dès 1853 en Californie on exploitait les gîtes aurifères alluvionnaires à la lance à incendie ; cette technique d'abattage hydraulique ayant l'avantage de pouvoir traiter de grandes quantités de matériaux en peu de temps, mais générant en aval un envasement des cours d'eau désastreux pour les fermiers utilisant l'eau pour l'irrigation. De la même façon, l'utilisation du mercure pour amalgamer l'or a conduit à répandre cette substance toxique dans les sols et dans beaucoup de cours d'eau de l'Ouest américain (Spitz K. \& Trudinger J., 2009).

9 Les sociétés minières industrielles ne se sont pas montrées plus précautionneuses que les prospecteurs artisanaux et ont généré des dégâts beaucoup plus considérables, à 
l'échelle des moyens techniques utilisés. Les gigantesques volumes de déblais générés par l'extraction, les résidus de traitement des minerais avec des solutions cyanurées constituent les principales menaces que fait peser l'exploitation minière industrielle sur l'environnement. L'exploitation minière est ainsi à l'origine de pollutions des eaux (figure 1) parfois très sévères sur près de $20000 \mathrm{~km}$ de cours d'eau de l'Ouest américain (Deshaies M., 2007).

Ces pollutions ont pu se produire pendant l'exploitation, par rejet parfois accidentel des solutions très acides filtrant à travers les gigantesques accumulations de minerai ou stockées dans les bassins de décantation à l'issue du processus de cyanuration. Ces rejets "accidentels » sont aussi souvent dus à l'insouciance, voire à l'absence de scrupules des exploitants miniers. C'est le cas notamment pour une des plus importantes catastrophes environnementales de l'histoire de l'exploitation minière aux États-Unis. Les bassins de décantation de la mine d'or et d'argent de Summitville au Colorado, située à $3500 \mathrm{~m}$ d'altitude, ont ainsi perdu en 1991-1992 plus de $320 \mathrm{~m}^{3} \mathrm{de}$ solutions cyanurées qui ont contaminé la rivière Alamosa, détruisant toute la faune sur $27 \mathrm{~km}$ de longueur (Berger A. 2002). Il est apparu que la société exploitant la mine depuis 1984, la Galactic Resources Limited, avait accumulé un volume énorme de substances toxiques $\left(600000 \mathrm{~m}^{3}\right)$ sans se donner les moyens de les traiter. La société s'est d'ailleurs déclarée en faillite en décembre 1991, laissant à l'État américain la charge du problème (Deshaies M., 2007).

11 Cependant, l'essentiel des pollutions constatées sur les cours d'eau de l'Ouest américain n'est pas dû à des rejets "accidentels ", mais résulte du drainage par les eaux souterraines d'anciennes mines abandonnées, car l'érosion des roches sulfurées exposées à l'air et à la pluie par les travaux miniers provoque une acidification très sensible des eaux. Selon une enquête partielle menée par le Mineral Policy Center (devenu en 2005 l'organisation Earthworks, www.earthworksaction.org) sur 32 États, il y aurait ainsi pas moins de 557650 mines abandonnées, dont 240000 dans l'Ouest intérieur (sans la Californie). Il est vrai aussi que ce recensement prend en compte non seulement les mines de métaux, mais aussi les mines de charbon, de matières minérales comme la potasse, ou même les carrières. Néanmoins, près de 15000 mines seraient à l'origine d'une pollution des eaux de surface ou des nappes phréatiques et 116000 poseraient des problèmes environnementaux. Une enquête fédérale menée en 1998 dans une partie des États de l'Ouest intérieur a ainsi détecté 32 sites miniers présentant un danger immédiat pour la santé (Deshaies M., 2007). 
Figure 1 : Les mines abandonnées dans l'Ouest américain et les pollutions par les métaux lourds

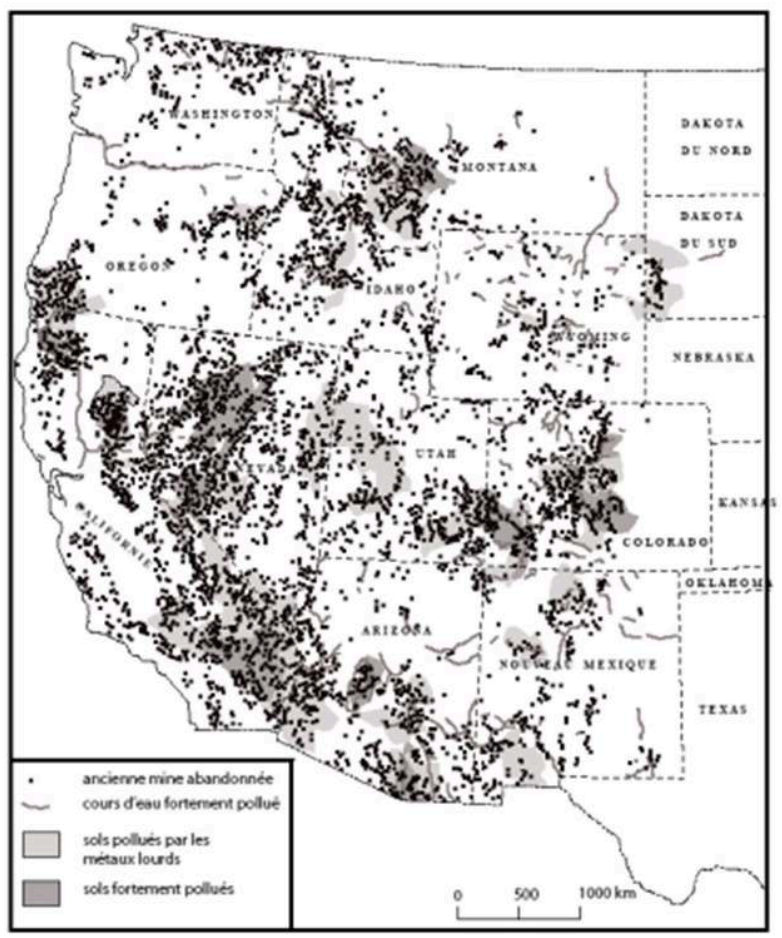

SOURCE : DESHAIES M., 2007

12 L'une des caractéristiques des paysages de l'Ouest américain ou des Andes est la multitude des grandes mines non réhabilitées qui forment de gigantesques cicatrices sur les flancs des montagnes. Beaucoup d'entre elles ne seront d'ailleurs probablement jamais réhabilitées car personne n'est en mesure de prendre en charge le coût financier démesuré que nécessiteraient de telles opérations. C'est le cas par exemple du Berkeley Pit, l'immense fosse creusée par l'exploitation du cuivre en bordure de la ville de Butte dans le Montana (photo 1). La découverte creusée par trente ans d'exploitation (de 1955 à 1982) atteint $500 \mathrm{~m}$ de profondeur et a été remplie en quelques années par un lac acide de $300 \mathrm{~m}$ de profondeur. En effet, la circulation des eaux à travers les roches a provoqué l'accumulation de substances toxiques telles que l'arsenic, le cadmium et l'acide sulfurique. Les risques que représente ce lac ont attiré l'attention en 1990 lorsque les cadavres de 342 oiseaux migrateurs ont été retrouvés à sa surface. Depuis lors, le Berkeley Pit et les immenses accumulations de déblais environnantes, classés site à risque environnemental majeur, sont l'objet de travaux de réhabilitation pris en charge par l'État fédéral dans le cadre du « Superfund » (Deshaies M., 2007). 
Photo 1 : Le Berkeley Pit à Butte (Montana) où, depuis l'arrêt de l'exploitation en 1982, s'est constitué un lac acide de $300 \mathrm{~m}$ de profondeur.

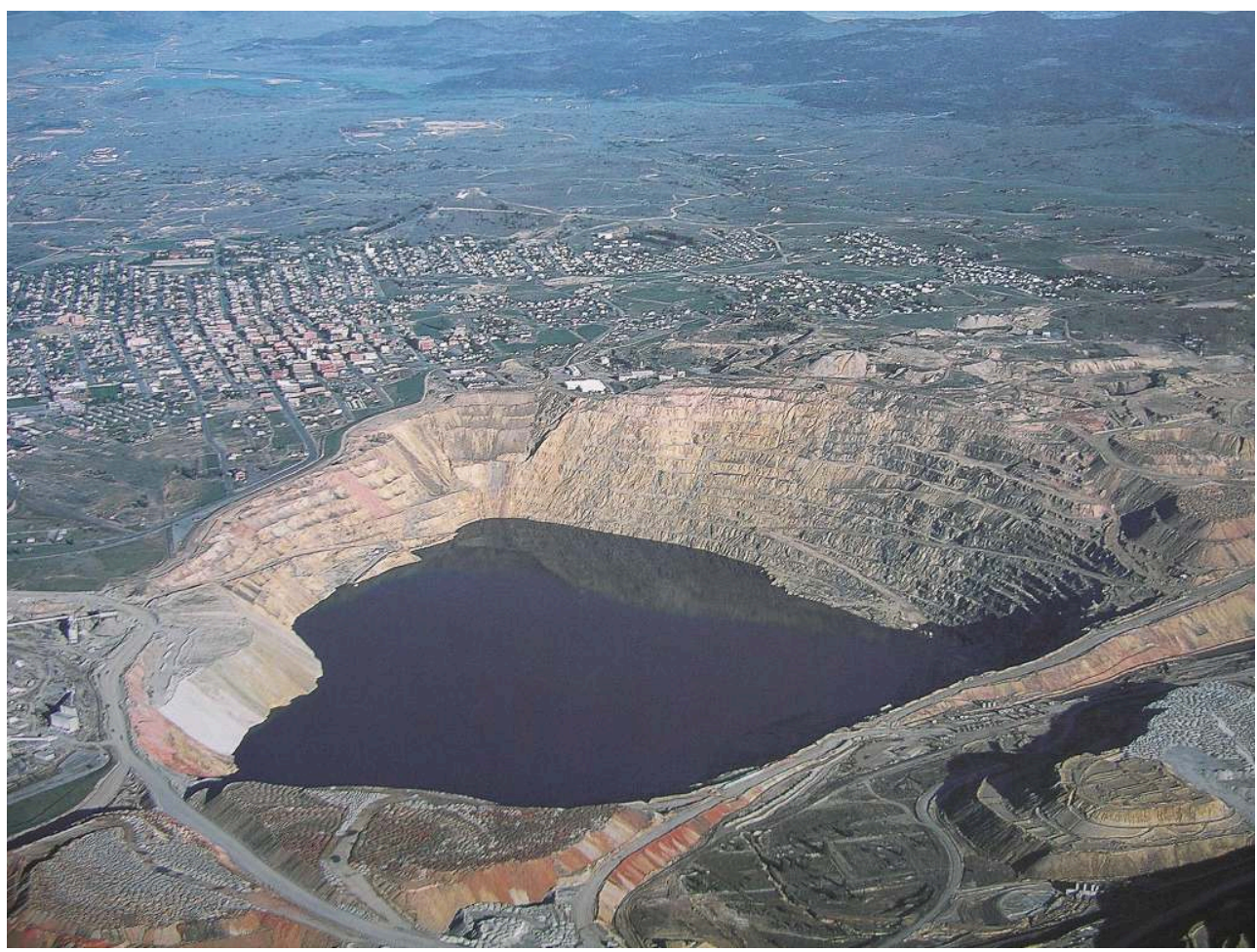

SOURCE : BERgER ALAN

\section{La nouvelle échelle d'une activité sous pression environnementaliste et sociétale}

\section{La multiplication des grands projets miniers}

À partir des années quatre-vingt et quatre-vingt-dix, l'exploitation minière connaît une nouvelle phase de développement qui concerne particulièrement les Amériques. Elle est portée principalement par l'essor rapide de la demande mondiale qui conduit à un doublement de la production d'or et d'argent et à un quadruplement de la production de la plupart des autres minerais métallifères entre 1970 et 2008. L'accroissement rapide de la demande, en lien avec l'essor des pays émergents comme la Chine, se traduit par une augmentation importante des prix des métaux à l'origine de l'accroissement des investissements dans l'exploitation minière (figure 2).

C'est dans ce contexte que les grandes exploitations à ciel ouvert vont se multiplier afin de permettre l'extraction des minerais les plus recherchés comme le cuivre, l'or ou l'argent dans des minerais à très faible teneur (moins de 5 grammes par tonnes d'or par exemple) comme au Nevada ou au Pérou. En effet, la forte augmentation des cours de l'or et de l'argent au cours des années soixante-dix assure la rentabilité des investissements considérables que nécessitent le creusement de grandes exploitations et le traitement par cyanuration sur une large échelle, de gigantesques volumes de minerais afin de récupérer des quantités limitées de métaux précieux. 
Les plus grandes mines d'or actuelles exploitent des minerais dont les teneurs moyennes n'excèdent pas 1 ou 2 grammes par tonne. Mais comme elles sont en mesure d'extraire plusieurs millions de tonnes de minerai par an, leur production s'élève à plusieurs dizaines de tonnes d'or pur par an. Ouverte au début des années 1990, la mine d'or de Yanacocha au Pérou, par exemple, a fourni jusqu'à 100 tonnes d'or par an et pour extraire cette quantité de métal, a déplacé environ 175 millions de tonnes de minerais et de stériles, soit environ trente fois le volume de la pyramide de Chéops (photo 2).

Figure 2 : Évolution de la valeur annuelle moyenne de l'or (en \$ par once) et de la production mondiale d'or (en dizaines de tonnes par an).

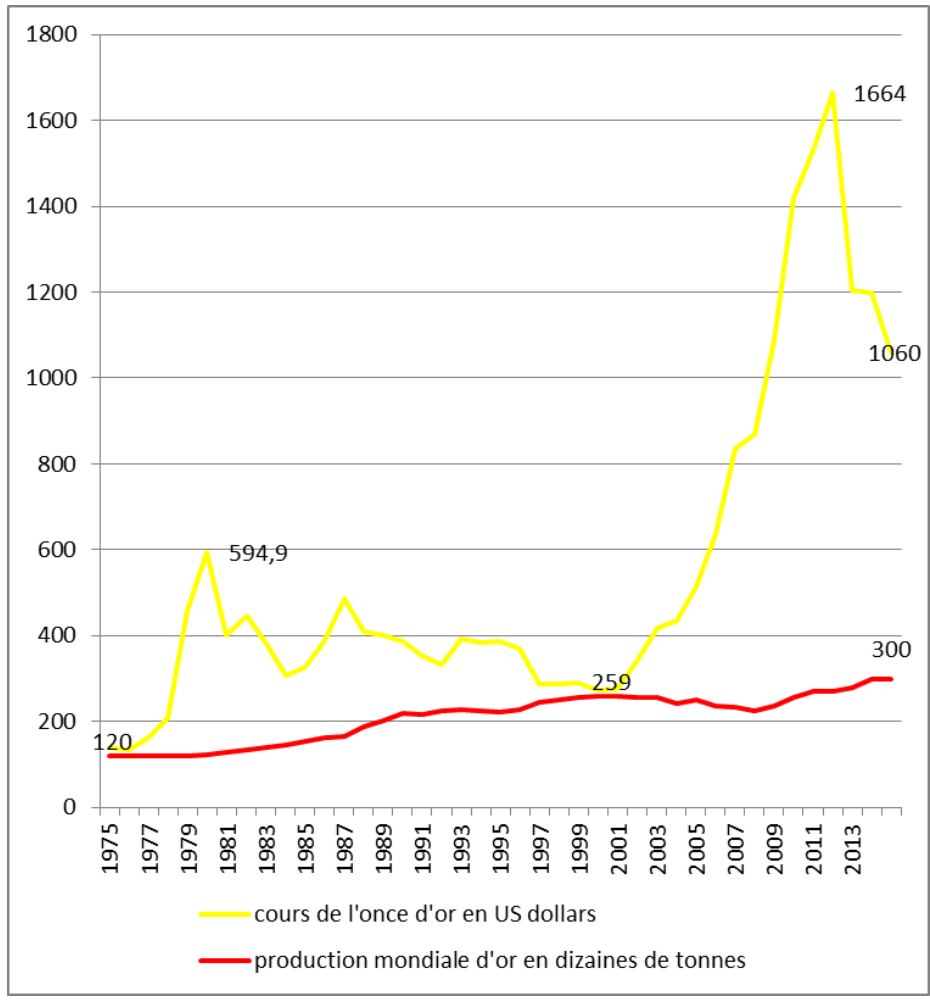

Sources: USGS pour la production mondiale d'or et http://onlygold.com/m/Prices/ Prices200Years.asp pour le cours annuel de l'or. 
Photo 2 : La mine de Yanacocha, près de Cajamarca au Pérou

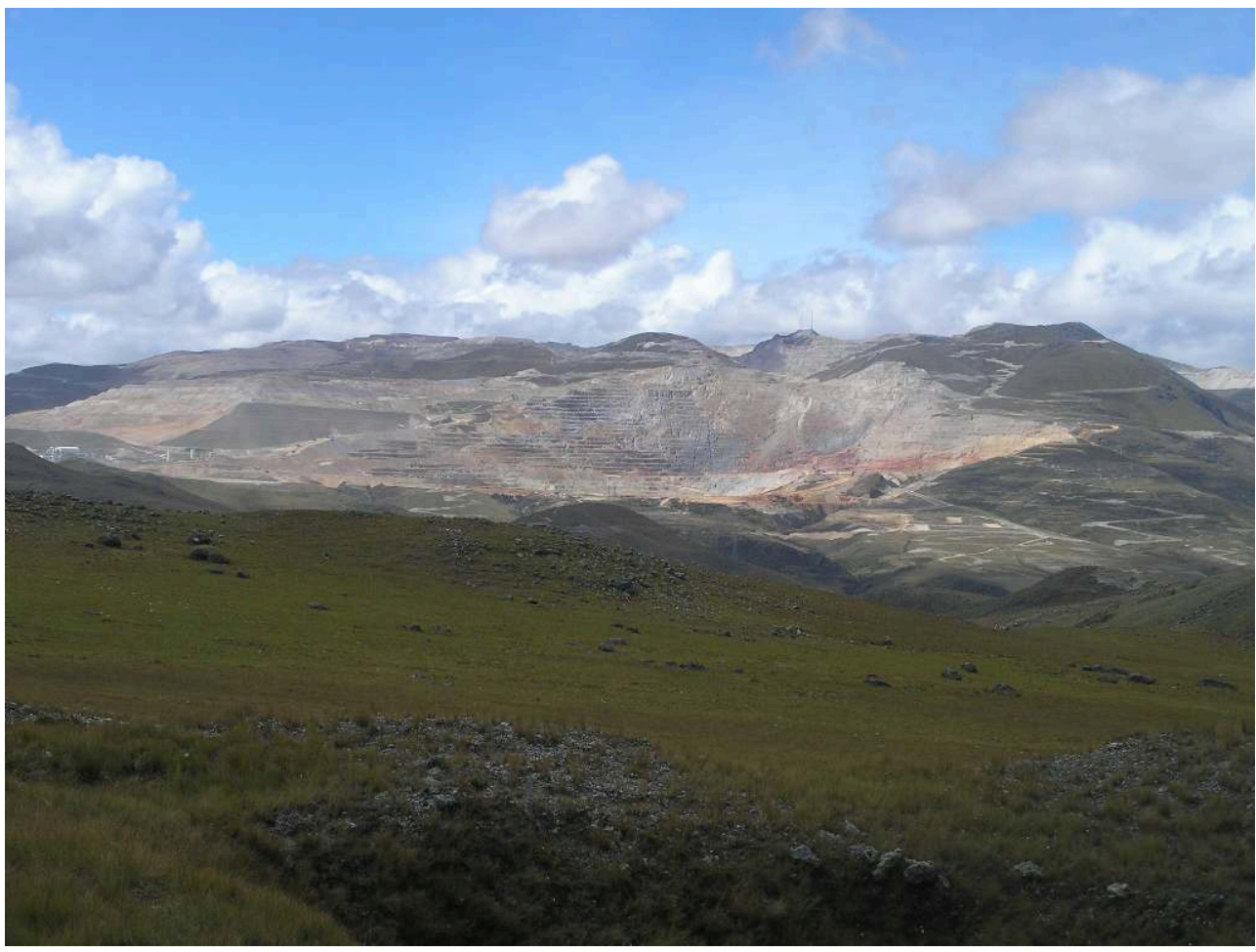

Source : Deshaies M., 2009

Figure 3 : Les conflits liés à des projets miniers en Amérique latine

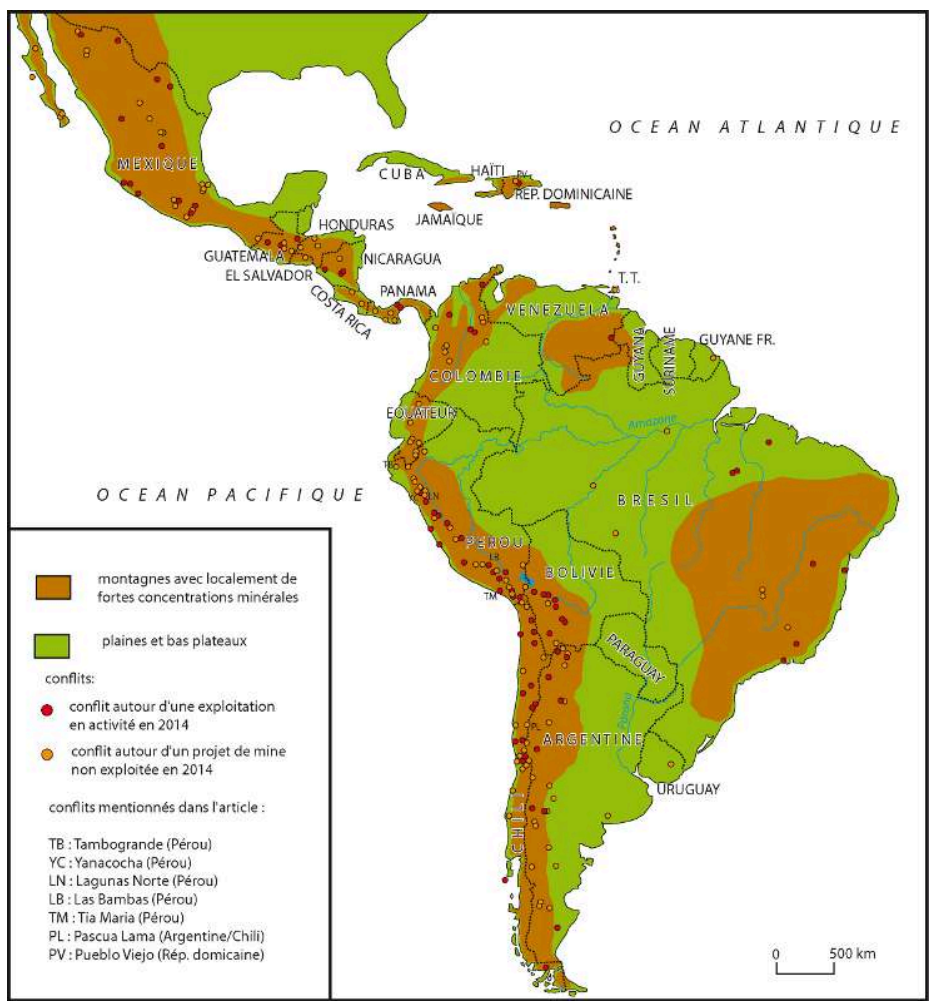

Source : http://www.conflictosmineros.net (source ModıfiéE) 
C'est dans ces conditions que les Amériques sont redevenues, dans les années quatrevingt-dix, le premier continent pour la production d'or, jusqu'alors dominée par l'Afrique du Sud. Alors qu'en 1980 les Amériques fournissaient tout juste 10\% de l'or extrait dans le monde (contre $56 \%$ pour la seule Afrique du Sud), depuis la fin des années quatre-vingt-dix leur part s'élève à $25-30 \%$ du total mondial (contre environ $15 \%$ pour la Chine, devenu le premier producteur mondial depuis 2006); ce qui est d'autant plus remarquable que, depuis 1997 on extrait en moyenne dans le monde deux fois plus d'or par an qu'en 1980 (figure 2). Cette renaissance des Eldorados s'explique d'abord par la fantastique croissance de la production d'or des États-Unis, passée de 30 tonnes en 1980 à 330 tonnes en 1992, pour atteindre un maximum de 366 tonnes en 1998 (Sources : USGS pour la production mondiale d'or et http://onlygold.com/m/ Prices/Prices200Years.asp pour le cours annuel de l'or). Les États-Unis qui étaient un producteur d'or très secondaire, avec seulement $2,5 \%$ du total mondial en 1980 , sont actuellement le quatrième producteur du monde (avec 211 tonnes en 2014), suivis par le Canada et le Pérou et derrière la Chine, l'Australie et la Russie. Depuis le début des années quatre-vingt-dix, leur part de la production mondiale est ainsi restée constamment comprise entre 8 et 15\%, même si après le maximum de 1998 elle a eu tendance à décliner. Néanmoins, on ne peut pas encore parler d'épuisement des gisements dans la mesure où les réserves prouvées d'or du sous-sol des Etats-Unis sont estimées par l'USGS (US Geological Survey, 2014) à environ 3000 tonnes, tandis que celles de l'ensemble des Amériques représentent environ un quart des réserves mondiales.

\section{La multiplication des oppositions et des conflits miniers}

L'accroissement de la demande mondiale et des prix des métaux a suscité la multiplication des projets miniers, non seulement dans des régions où se trouvaient des gisements connus de longue date, mais aussi dans de nouvelles régions où ont été découverts des gisements à faible teneur dont l'exploitation n'était autrefois pas rentable. Tous les pays ayant connu un essor de l'exploitation minière depuis les années 1990 ont vu ainsi se multiplier les conflits autour de projets considérés comme menaçants pour leur cadre de vie par les populations locales, Ces conflits sont particulièrement nombreux en Amérique latine où beaucoup de projets miniers ont été lancés par des compagnies étrangères, le plus souvent originaires du Canada, ou des États-Unis, et plus récemment de Chine. Cela contribue à l'exacerbation des conflits car les compagnies étrangères sont accusées par les opposants aux mines d'extraire des richesses qui ne profitent pas aux communautés locales dont l'environnement et l'économie agricoles risquent d'être détruits par les pollutions issues de l'exploitation minière.

L'observatoire des conflits miniers en Amérique latine ${ }^{1}$ qui rassemble de nombreuses ONG en a recensé 217 en 2014 dans 19 pays. Le Pérou (39), le Mexique (37) et le Chili (36) représentent à eux seuls la moitié des conflits (figure 3). Cette situation de forte conflictualité est une conséquence directe de la multiplication des investissements dans les territoires des communautés indigènes de ces différents pays. Le Pérou constitue un exemple emblématique. La forte croissance de la production minière du Pérou, redevenu un Eldorado, et notamment le premier producteur du monde d'argent et le sixième producteur d'or, est liée à la mise en valeur de nouveaux gisements à faible 
teneur sous la forme de très grandes exploitations à ciel ouvert, comme celle de Yanacocha, près de Cajamarca, dans le nord du pays. Au Pérou, près de $90 \%$ des surfaces ayant fait l'objet de concessions minières l'ont été depuis 1990 et pour l'essentiel après 2000. En 2009, les concessions minières couvraient près de $14000 \mathrm{~km} 2$, soit $11 \%$ de la superficie du pays.

Les conflits autour des projets d'exploitation minière, ou d'extraction d'hydrocarbures, ont très souvent donné lieu à des affrontements au cours desquels pas moins de 53 personnes ont été tuées au Pérou depuis 2011. Les conflits autour de l'extension de la mine d'or de Yanacocha, de celle de Lagunas Norte, de la mine de cuivre et d'or de Tía María, de la mine de cuivre de Las Bambas (figure 3), ou du projet de creuser une nouvelle mine d'or à Tambo Grande dans le nord du pays (province de Piura), sont emblématiques de cette pression environnementaliste qui pèse sur les sociétés minières (Deshaies M., 2007 ; Deshaies M. \& Baudelle G., 2013). Pendant toute la durée de leur exploitation, Yanacocha et Lagunas Norte ont été accusées de polluer les ressources en eau locales; si bien que les compagnies minières ont dû faire face, à de nombreuses reprises, à des manifestations ou à des opérations de blocage des sites miniers. Le projet d'exploitation de l'or de Tambo Grande déposé en 1999 par la société canadienne Manhattan Minerals a même dû être abandonné à la suite de l'opposition des populations locales qui craignaient que le creusement d'une grande découverte ne provoque un assèchement partiel du fleuve Piura sur lequel reposait leur agriculture irriguée et que l'exploitation minière ne génère des rejets massifs de substances toxiques ruinant leur activité. La société mexicaine à l'origine du projet minier de Tía María a dû pour sa part investir dans une installation supplémentaire de désalinisation afin de répondre à l'inquiétude des agriculteurs locaux, dont les manifestations hostiles se sont soldées par un bilan de trois morts. L'ouverture de la mine de cuivre de Las Bambas en décembre 2015, opérée par la compagnie chinoise MMG, a été précédée de violentes manifestations, faisant quatre morts parmi les opposants qui exigeaient la démolition de l'installation de traitement du minerai. Selon eux, celle-ci menacerait de polluer le rio Chalhuahuacho dont l'eau est vitale pour l'agriculture d'une partie de la région d'Apurimac.

À cette opposition radicale aux risques de pollution des eaux par les mines, s'ajoute une critique plus fondamentale sur les conséquences de la trop forte dépendance du développement économique de ces pays à l'égard des ressources minières. En effet, beaucoup de pays du Tiers-monde possédant d'importantes ressources minières ou énergétiques ont connu un développement moins important que les pays dépourvus de ressources du sous-sol (Gylfason T., 2001). Cette thèse de la malédiction des ressources (Resource Curse) a été beaucoup argumentée par tout un courant d'économistes (par exemple Sachs J. \& Warner A., 1995, 1997, 2001 ; Auty R., 1993 et 2001) qui se sont efforcés de montrer par quels mécanismes l'exploitation de ressources minérales abondantes pouvait avoir une influence négative sur le développement économique.

21 Même si cette "malédiction des ressources" n'est pas nécessairement inévitable (Wright, G., 1990 ; Findlay, R. \& Lundahl, M., 1999 ; Wright, G. \& Czelusta, J. 2004 ; Jebrak, M. 2015), cette théorie apporte néanmoins un argument supplémentaire aux ONG et aux populations qu'elles soutiennent dans leur combat contre les projets miniers. Pour les militants opposés à l'exploitation minière, celle-ci détruit l'environnement sans permettre le développement socio-économique. En conséquence, il vaut mieux l'interdire. 
Certains conflits opposant les communautés locales aux sociétés minières ont reçu ainsi un important écho médiatique et les ONG environnementalistes ont développé des campagnes visant à attirer l'attention de l'opinion publique sur les nuisances environnementales et sociales des grands projets miniers. L'exploitation de l'or est particulièrement visée et depuis 2004, une campagne intitulée « No Dirty Gold " est organisée aux États-Unis par les ONG Earthworks et Oxfam America afin de responsabiliser les consommateurs et les fabricants d'objets utilisant de l'or (http:// www.nodirtygold.org). Avec le slogan «plus vous en saurez sur l'or, moins il brillera ", elles essaient de responsabiliser les consommateurs et il est demandé aux joailliers, aux fabricants d'électronique et autres industries de s'engager à ne pas utiliser d'or provenant d'exploitations minières ayant un impact négatif pour les populations locales et l'environnement. Au cours des dernières années, les initiatives (Fairtrade Gold $^{2}$, Conflict-Free Gold ${ }^{3}$, etc) visant à mettre en place des certifications attestant l'origine de l'or d'exploitations minières qualifiées d'« environnementalement et socialement responsables » se sont multipliées.

\section{Les entreprises minières face aux défis environnementaux et sociétaux}

\section{La nouvelle stratégie des compagnies minières face à une pression sociale croissante}

23 Avec la multiplication des conflits autour des projets miniers au début du XXIe siècle, les compagnies ont été soumises à une pression croissante, non seulement dans les pays où sont extraits les minerais, mais aussi dans ceux vers lesquels ils sont exportés. Cette pression s'est exprimée à travers les recommandations de différentes institutions internationales. En 2002, le programme des Nations Unies pour l'environnement (United Nations Environment Programme - UNEP) a ainsi insisté sur la nécessité pour les compagnies minières de prendre en compte la gestion de l'environnement et d'ouvrir le dialogue avec les communautés d'habitants directement concernées par leurs projets. Les grandes banques internationales ont défini en 2003 les «principes de l'Équateur » qui soumettent le financement des projets miniers au respect d'un certain nombre de critères sociaux et environnementaux. En 2008, la International Finance Corporation - IFC (la Société Financière Internationale), l'ONU et l'OCDE ont proposé aux compagnies minières de respecter un certain nombre de recommandations en matière environnementale et de relation avec les communautés (Jebrak, M., 2015).

De leur côté, les compagnies minières ont répondu à ces pressions insistantes en montrant qu'elles avaient intégré les préoccupations sociales et environnementales qui s'étaient exprimées depuis une vingtaine d'années. À l'occasion du sommet sur le développement durable, qui s'est tenu en Afrique du Sud en 2002, neuf des plus grandes entreprises minières du monde ont fait rédiger par l'Institut International pour l'environnement et le développement (International Institute for Environment and Development (I.I.E.D), le rapport Breaking New Ground: Mining, Minerals and Sustainable Development ${ }^{4}$. L'objectif de ce rapport était de montrer qu'il n'y avait pas lieu de diaboliser l'industrie minière, en répondant point par point aux critiques formulées par les ONG environnementalistes à l'encontre des grandes compagnies minières. Le rapport définissait les principes d'une exploitation responsable, favorisant 
l'amélioration de la situation économique des communautés locales (Spitz K. et Trudinger J., 2009).

Les compagnies minières ont ainsi progressivement développé toute une stratégie visant à obtenir une « licence sociale d'opération » (social license to operate) ; autrement dit disposer d'une autorisation d'exploiter accordée par la communauté directement concernée (Thomson I. \& Boutilier R., 2011 ; Jebrak M., 2015). Les grandes compagnies minières ont en effet compris que la réussite de leurs projets reposait de plus en plus sur l'obtention d'une acceptation de leur activité par les communautés affectées ; les conflits miniers pouvant rapidement ternir leur image et même leur coûter très cher, avec dans le pire des cas une annulation du projet. De nombreuses entreprises se sont ainsi engagées dans une démarche visant, dès la phase d'exploration, à ouvrir le dialogue avec les communautés afin de répondre par avance à leurs inquiétudes et à leurs attentes. Elles se sont ainsi dotées de personnels dédiés à la communication avec les communautés, afin d'obtenir, dans un premier temps, une légitimité, puis dans un second temps, d'établir des liens de confiance (Thomson I. \& Boutilier R., 2011). La prise en compte des attentes de la communauté au cours même de la construction de la mine est un processus cependant complexe, dans la mesure où le projet lui-même peut fortement évoluer en fonction même des résultats de l'exploration. D'où la nécessité d'un dialogue dans la durée, dans la mesure où les impacts de la future mine ne peuvent pas être précisés dès le départ. En effet, tout dépend des caractéristiques de l'exploitation minière qui sera finalement décidée (ou non) en fonction des critères économiques et géologiques.

\section{Des principes à la pratique du développement durable par les compagnies minières}

26 L'obtention de la licence sociale d'opération par une compagnie minière repose sur sa capacité à obtenir la confiance des communautés et donc à pouvoir attester son engagement dans une exploitation socialement responsable sur le plan environnemental. Cela suppose que les caractéristiques du projet minier puissent satisfaire plus ou moins complètement aux trois piliers du développement durable : un impact maîtrisé sur l'environnement, favoriser le développement économique de la communauté locale et lui apporter des bénéfices sociaux supérieurs aux nuisances qu'il génère.

Évidemment, l'évaluation des différents impacts d'un projet minier est pour le moins compliquée et ne peut que difficilement déboucher sur un consensus, entre ceux qui accordent une valeur sacrée à l'environnement et ceux qui considèrent qu'une exploitation maîtrisée est possible et même nécessaire pour permettre le développement des communautés. C'est pourquoi les compagnies minières s'attachent à montrer, par des réalisations concrètes, que leurs activités peuvent apporter un bénéfice tangible aux communautés concernées. Dans ce domaine, il est clair que toutes les compagnies minières ne peuvent pas se prévaloir de la même crédibilité. Ce sont surtout les grandes compagnies minières qui peuvent appuyer leur communication sur des projets déjà réalisés avec des actions de soutien aux communautés locales et de réhabilitation environnementale. Ces actions exposées dans des bulletins et des rapports tels que "Beyond borders ${ }^{5}$ " de Barrick Gold Corporation et «Beyond the Mine $^{6} »$ de Newmont Mining Corporation comportent généralement trois volets : la 
responsabilité environnementale de la compagnie dans ses techniques d'exploitation, sa responsabilité sociale à travers les actions menées en faveur des communautés, son engagement dans des actions de restauration ou d'amélioration de l'environnement (Spitz K. \& Trudinger J., 2009).

- Les compagnies s'efforcent de montrer leur responsabilité environnementale en utilisant les techniques les plus modernes et les moins agressives pour l'environnement. Dans certains cas, elles avancent qu'elles sont même susceptibles de contribuer à l'amélioration de la situation, notamment de la qualité des eaux qui est toujours l'un des grands problèmes des régions minières (Spitz K. \& Trudinger J., 2009). Afin de pouvoir développer son projet de Pueblo Viejo en République dominicaine, la Barrick Gold a signé en novembre 2009 un accord avec le gouvernement dominicain dans lequel elle s'engageait à prendre en charge les frais de dépollution des anciens sites miniers dans le périmètre de sa concession, ce qui a permis de solder l'héritage de plusieurs décennies d'exploitation prédatrice. Pour la mine de Yanacocha qui a été accusée de polluer les eaux de la région et notamment celles utilisées par l'agriculture et la ville de Cajamarca au Pérou, la Newmont a dû investir des sommes importantes dans des bassins destinés à piéger les sédiments et les polluants qui pourraient provenir des travaux miniers.

- La responsabilité sociale est illustrée par le soutien à des projets de commercialisation de produits locaux qui permettent d'apporter de nouvelles ressources financières aux communautés. Les compagnies minières ont aussi initié des projets de coopération et d'aide aux communautés, en construisant des écoles et en finançant des programmes d'éducation et de soin, assurant ainsi des services publics que certains États ne peuvent pas remplir. L'objectif est de construire une relation de confiance avec les populations locales. L'aide multiforme aux communautés est un volet essentiel de la stratégie des compagnies minières qui y consacrent des moyens financiers substantiels. Cette aide peut aussi se matérialiser par des travaux d'infrastructure permettant de développer l'économie locale. C'est le cas par exemple à Yanacocha où, depuis 2006, l'ancienne mine à ciel ouvert de San José a été transformée en réservoir de stockage de l'eau pour alimenter des canaux d'irrigation utilisés par plusieurs milliers d'agriculteurs locaux. Cette participation de la compagnie minière au développement de l'agriculture irriguée dans une région à longue saison sèche est particulièrement mise en avant, dans la mesure où ce projet permet de prendre à contrepied l'argumentation des opposants à la mine qui l'accusent de polluer les ressources locales en eau.

- Les compagnies minières essaient aussi de se construire une image «verte » en finançant des projets de protection ou de restauration de la nature, de reboisement des zones minières, en réhabilitant d'anciennes mines, ou en investissant dans les énergies renouvelables.

28 Les grandes compagnies minières ont en effet compris que la réussite de leurs projets reposait de plus en plus sur l'obtention d'une acceptation de leur activité par les communautés affectées; les conflits miniers pouvant rapidement ternir leur image, non seulement dans le pays où sont extraits les minerais, mais aussi dans ceux vers lesquels ils sont exportés. Aussi, pour se rendre acceptables, elles montrent dans quelle mesure leurs activités peuvent aussi contribuer au développement durable des territoires (Spitz, K. \& Trudinger, J., 2009) :

Ensuite, elles s'efforcent de montrer les répercussions positives de leur activité en termes économiques et sociaux pour les populations locales. Les compagnies minières soutiennent par exemple des projets de commercialisation de produits locaux qui 
permettent d'apporter de nouvelles ressources financières aux communautés. Les compagnies minières ont aussi initié des projets de coopération et d'aide aux communautés, en construisant des écoles et en finançant des programmes d'éducation et de soin, assurant ainsi des services publics que certains États ne peuvent pas remplir. Elles veulent ainsi obtenir le soutien des populations locales et afficher leur contribution au développement des communautés. L'aide multiforme aux communautés est un volet essentiel de la stratégie des compagnies minières qui y consacrent des moyens financiers substantiels :

- La réhabilitation des mines après leur fermeture: à travers les exemples de mines actuellement fermées et en cours de réhabilitation, les compagnies minières ont la possibilité de montrer qu'elles sont capables de reconstruire un environnement acceptable, voire même de qualité après la fin de l'exploitation. Pour répondre aux critiques nombreuses qu'a suscitées la mine de Yanacocha et prévenir ainsi de nouveaux conflits qui pourraient entraver le développement du projet de Conga, la Newmont a préparé un plan de clôture visant à réhabiliter la mine après l'arrêt des opérations d'extraction prévu en $2018^{7}$. Après une période de démantèlement des infrastructures minières et de remodelage des découvertes qui devrait s'étaler sur sept ans, il est prévu de mettre en place un système de traitement des eaux devant prévenir les risques de pollution en métaux lourds sur les cours d'eau de la région. Cela dit, ces actions n'ont pas empêché la population locale de manifester violemment contre le projet Conga qui, depuis août 2012, est suspendu par décision du gouvernement péruvien.

- Le développement de projets de reboisement (photo 3): la Newmont a financé le reboisement de plusieurs milliers d'hectares autour de la mine de Yanacocha, reconstituant ainsi des forêts détruites depuis longtemps par la surexploitation des ressources en bois. De la même manière, dans le cadre de la réhabilitation des anciennes exploitations minières de Pueblo Viejo, la Barrick Gold a développé depuis 2008 un plan de décontamination des sols et de reforestation en replantant plus de 20000 arbres par mois.

- Le financement des énergies renouvelables: c'est une stratégie qui s'inscrit dans les préoccupations concernant la réduction des émissions de gaz à effet de serre. Les activités minières dont les énormes machines et camions sont de gros consommateurs de produits pétroliers, cherchent donc aussi à verdir leur image en investissant dans le secteur très emblématique des énergies renouvelables. Dans le cadre de la promotion du projet de Pascua-Lama, la Barrick Gold a installé une grande éolienne pour produire une partie de l'électricité de sa mine de Veladero à plus de $4000 \mathrm{~m}$ d'altitude. Elle a également financé un grand parc éolien pour un montant de 50 millions de dollars à la Higuera dans la région de Coquimbo. Cependant, si ces projets sont présentés comme devant fournir une partie de l'approvisionnement électrique de la mine, ils apparaissent surtout comme des instruments de communication destinés à illustrer la responsabilité environnementale de la société. Ces efforts n'ont jusqu'à présent pas suffi pour permettre à la compagnie de surmonter toutes les résistances à son projet minier, accusé par ses détracteurs de menacer la qualité des ressources en eau provenant de glaciers locaux devant être affectés par l'exploitation. Depuis octobre 2013, la Barrick Gold a annoncé la suspension du projet, motivée aussi en partie par la baisse des prix de l'or. 


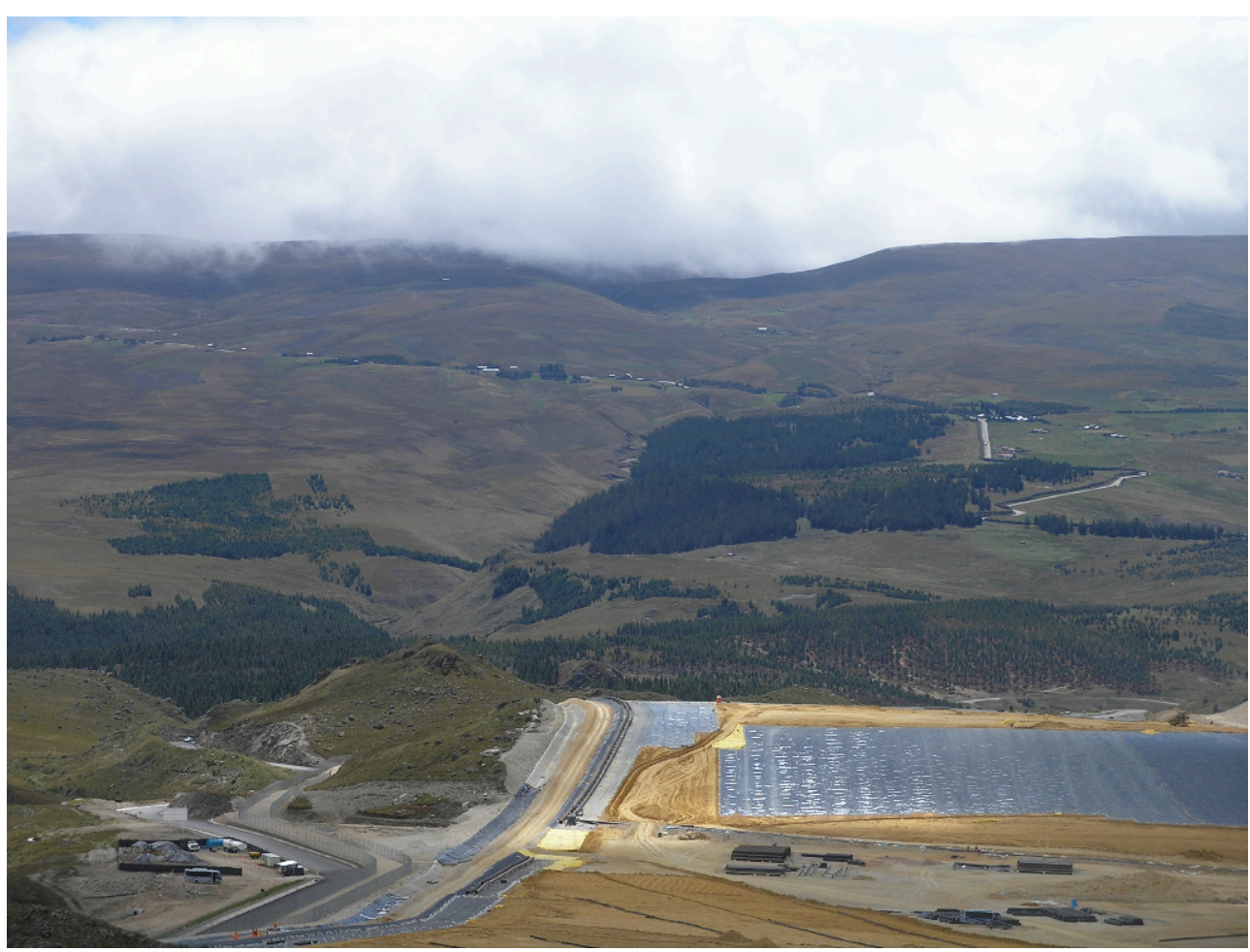

Source : Deshaies M., 2009

\section{Conclusion}

Longtemps perçue comme source d'enrichissement, l'exploitation minière est de plus en plus considérée d'abord comme une menace pour l'environnement. L'exploitation prédatrice qui a longtemps dominé, multipliant les paysages dégradés et polluant des milliers de kilomètres de cours d'eau a beaucoup contribué à justifier cette perception ; si bien que désormais le mythe de l'Eldorado ne suffit plus à faire accepter les grands projets d'exploitation minière. Ces réactions traduisent une évolution majeure dans les relations entre l'activité minière et les populations locales. Né en Amérique du Nord, le courant environnementaliste a aussi une forte audience en Amérique du Sud où les sociétés minières nord-américaines ont beaucoup investi au cours des années quatrevingt-dix. Il est vrai en effet que l'accroissement considérable de la production minière en Amérique Latine depuis les années quatre-vingt est dû principalement à l'ouverture de nouvelles mines à ciel ouvert, de taille souvent gigantesque, dans des régions jusqu'alors peu ou pas touchées par cette activité.

31 Aussi, le développement de nouveaux grands projets d'exploitation minière se heurte-til de plus en plus souvent à une opposition des populations locales soutenues par les ONG environnementalistes. Celles-ci soulignent, entre autres, l'incompatibilité entre exploitation minière et développement durable et tendent à diaboliser l'activité des grandes sociétés minières nord-américaines qui doivent déployer des efforts de plus en plus importants de communication auprès des populations locales et satisfaire à des exigences environnementales croissantes. La stratégie des sociétés minières consiste à établir des relations de confiance avec les communautés locales afin d'obtenir une 
«licence sociale d'opération». L'établissement de ces relations suppose que la compagnie minière puisse démontrer que son projet aura un impact maîtrisé sur l'environnement et pourra favoriser le développement économique de la communauté en lui apportant des bénéfices sociaux supérieurs aux nuisances qu'il génère. Si les plus grandes compagnies minières peuvent appuyer leur argumentation sur de nombreux projets déjà réalisés, cela est loin de suffire à leur garantir le succès comme le montre la suspension du projet de la Barrick Gold à Pascua Lama, ou de la Newmont à Conga.

L'un des paradoxes de la grande exploitation minière contemporaine est qu'elle suscite une opposition croissante, alors qu'elle n'a jamais fait autant d'efforts pour réduire son impact sur l'environnement. La pression environnementaliste a ainsi contribué à limiter les nuisances créées pendant la durée d'activité de la grande exploitation minière industrielle; si bien que, sauf accident, celle-ci génère au total moins de pollutions que l'exploitation minière artisanale clandestine sévissant un peu partout dans les pays en voie de développement. Il n'en reste pas moins vrai que du fait même de son échelle, la grande exploitation minière laisse dans les paysages des héritages d'une telle dimension, que leur réhabilitation apparait comme extrêmement problématique.

La question du devenir des territoires et des populations après l'arrêt de l'exploitation minière est en conséquence le problème le plus épineux et le moins évoqué par les compagnies minières. Même dans l'hypothèse où les pollutions auraient pu être complètement maîtrisées durant la période d'activité de la mine, les transformations des paysages et plus largement du cadre de vie des populations sont tellement importantes que la capacité de résilience du territoire est profondément altérée. Pour gagner en crédibilité, les compagnies minières doivent aussi travailler cette question du devenir du territoire exploité après la fermeture de la mine. Cela est d'autant plus une nécessité que la plupart des projets miniers actuels ont une durée de vie relativement courte, une à deux décennies seulement.

\section{BIBLIOGRAPHIE}

Auty, Richard, Sustaining Development in Mineral Economies: The Resource Curse Thesis, London, Routledge 1993.

Auty, Richard, Resource Abundance and Economic Development, Oxford, Oxford University Press, 2001.

Bebbington, Anthony, et al, "Contention and Ambiguity: Mining and the Possibilities of Development”, Brooks World Poverty Institute Working Paper No. 57, Manchester, Brooks World Poverty Institute, 2008.

Bebbington, Anthony, et Mark Williams, "Water and mining conflicts in Peru”, Mountain Research and Development, Vol 28 No 3/4 Aug-Nov 2008, p. 190-195. Disponible en ligne: http:// snobear.colorado.edu/Markw/Research/08_peru.pdf. Page consultée le 30 juin 2014. 
Bebbington, Anthony, Bury, Jeffrey, Institutional challenges for mining and sustainability in Peru, sous la direction de B. L. Turner II, Arizona State University, Tempe, AZ, Disponible en ligne : http://www.pnas.org/content/early/2009/09/23/0906057106.full.pdf+html. Page consultée le 30 juin 2014.

Berger, Alan, Reclaiming the American West, Princeton Architectural Press, New York, 2002.

Blaikie, Piers, "Understanding environmental issues", dans Morse, Stephen et Michael Stocking (dir.), People and Environment, University of East Anglia, School of Developing Studies Norwich, 1995, p. 1-30.

Coulson, Michael, The History of Mining, Harriman House Ltd, Petersfield, 2012.

Davis, Graham et John Tilton, Should Developing Countries Renounce Mining? A Perspective on the Debate, Denver, Colorado, Colorado School of Mines, 2002.

Deshaies, Michel, Les territoires miniers, exploitation et reconquête, Paris, Ellipses, 2007.

Deshaies, Michel, « Grands projets d'exploitation minière et stratégie des firmes pour se rendre environnementalement acceptables », L'Espace Politique, vol. 15, numéro 3. Disponible en ligne : https://espacepolitique.revues.org/2113. Page consultée le 11 août 2014.

Deshaies, Michel et Guy Baudelle, Ressources naturelles et peuplement, Ellipses, 2013.

Findlay, Ronald et Mats Lundahl, Resource-Led Growth - A Long-Term Perspective. The Relevance of the 1870-1914 Experience for Today's Developing Economies, UNU World Institute for Development Economics Research (UNU/WIDER). Working Paper Nr162.

Gylfason Thorvaldur, Natural resources, education, and economic development, European Economic Review, vol. 45, 2001, p. 847-859.

International Institute for Environment and Development (I.I.E.D), Breaking New Ground: Mining, Minerals and Sustainable Development, 2002, http://www.iied.org/mmsd-final-report. Page consultée le 5 décembre 2016.

Jebrak, Michel, Quels métaux pour demain? Les enjeux des ressources minérales, Dunod, 2015.

McMahon, Gary et Rémy Felix, Large Mines and the Community: Socioeconomic and Environmental Effects in Latin America, Canada, and Spain. Toronto, World Bank, IDRC, 2001.

Sachs, Jeffrey et Andrew Warner, “The Big Push, Natural Resource Booms and Growth”, Journal of Development Economics, vol. 59, n 1, 1999, p. 43-76.

Sachs, Jeffrey et Andrew Warner, Natural Resource Abundance and Economic Growth, HIID Working Paper, Novembre 1997.

Sachs, Jeffrey et Andrew Warner Andrew, Natural Resource Abundance and Economic Growth, NBER Working Paper 5398. Cambridge, Mass., National Bureau of Economic Research, 1995.

Sachs, Jeffrey et Andrew Warner, "Natural Resources and Economic Development: The curse of natural resources", European Economic Review, vol. 45, 2001, p. 827-838

Spitz, Karlheinz et Trudinger John, Mining and the environment, Boca Raton, CRC Press, 2009.

Thomson, Ian et Rober Boutilier, "Social license to operate" dans Peter Darling (dir.), SME Mining Engineering Handbook, Littleton, CO, Society for Mining, Metallurgy and Exploration, 2011, p. 1779-1796. 
US Geological Survey, Mineral Commodity Summaries, Reston, Virginia, US Department of the Interior, 2014.

Wright, Gavin, “The Origins of American Industrial Success, 1879-1940”, The American Economic Review, Vol. 80, nº 4, sept. 1990, p. 651-678.

Wright, Gavin et Jesse Czelusta, "The Myth of the Resource Curse", Challenge, vol. 47, numéro 2, mars/avril 2004, p. 6-38.

\section{NOTES}

1. http://www.conflictosmineros.net/

2. http://www.fairtrade.org.uk/en/farmers-and-workers/gold

3. http://www.gold.org/gold-mining/responsible-mining/conflict-free

4. http://www.iied.org/mmsd-final-report

5. http://barrickbeyondborders.com/

6. http://sustainabilityreport.newmont.com/2015/

7. http://www.yanacocha.com/

\section{RÉSUMÉS}

La richesse minière des Amériques a joué un rôle essentiel dans la diffusion du peuplement dans les régions les plus hostiles du continent. L'exploitation des nombreux gisements métallifères découverts depuis le XVIe siècle a revêtu longtemps un caractère prédateur et dévastateur pour l'environnement. Il en reste de nombreux héritages sous forme de puits et de galeries de mines, ou de vastes exploitations à ciel ouvert abandonnées, à l'origine d'une forte pollution des eaux. Cette richesse minière est aujourd'hui encore une réalité, puisque plus de la moitié de la valeur de la production mondiale de minerais vient du Nouveau Monde. La forte croissance de l'extraction depuis les années soixante-dix a été rendue possible par le développement de l'exploitation de nouveaux gisements à ciel ouvert. Ces nouvelles mines ont un impact paysager sans précédent à l'origine d'une montée des oppositions et d'une augmentation des conflits avec les communautés locales craignant des conséquences environnementales négatives. Sous la pression des communautés d'habitants et des mouvements écologistes, les grandes sociétés minières ont dû progressivement réduire l'impact environnemental de l'exploitation et s'engager dans des actions visant à favoriser l'acceptabilité de leur activité.

The mineral wealth of the Americas has played a pivotal role in disseminating the settlements stand in the most hostile regions of the continent. The exploitation of many ore deposits discovered since the sixteenth century has long taken a devastating, predatory character to the environment. It left many legacies such as well and and mine galleries, or large, abandoned opencasts, which are at the origin of a significant water pollution.

This mineral wealth is still a reality today, as more than half the value of global ore production comes from the New World. The strong growth in mining since the seventies has been made possible by the development of the exploitation of new open pit deposits. These new mines have an unprecedented impact on the landscape and have given rise to unprecedented opposition and 
increasing conflict with local communities fearing the negative environmental consequences. Under pressure from community residents and environmental groups, large mining companies gradually had to reduce the environmental impact of their operations and engage in actions to promote the acceptability of their activities.

La riqueza minera de las Américas ha jugado un papel fundamental en la difusión del poblamiento en las regiones más hostiles del continente. La explotación de numerosos yacimientos metalíferos descubiertos desde el siglo XVI ha revestido un carácter depredador y devastador para el medio ambiente. Quedan numerosas herencias, bajo la forma de pozos y de galerías de mina o de extensas explotaciones a cielo abierto abandonadas, que originan de una fuerte contaminación de las aguas.

Esta riqueza minera aún sigue siendo hoy en día una realidad, pues más de la mitad del valor de la producción mundial de minerales proviene del Nuevo Mundo. El fuerte crecimiento de la extracción desde los años setenta ha sido posible gracias al desarrollo de la explotación de nuevos yacimientos a cielo abierto. Estas nuevas minas tienen un impacto paisajístico sin precedentes, siendo origen de una creciente oposición y de un aumento de los conflictos con las comunidades locales, que temen las consecuencias medioambientales negativas. Bajo la presión de las comunidades de habitantes y de los movimientos ecologistas, las grandes sociedades mineras han debido reducir progresivamente el impacto medioambiental de la explotación y comprometerse a realizar acciones encaminadas a favorecer la aceptabilidad de su actividad.

\section{INDEX}

Mots-clés : mine, environnement, conflit, acceptabilité sociale, responsabilité environnementale, réhabilitation

Palabras claves : mina, cielo abierto, medio ambiente, conflicto, aceptabilidad social, responsabilidad medioambiental, reconstrucción ambiental

Keywords : mine, environment, conflict, social acceptability, social responsibility, remediation

\section{AUTEUR}

\section{MICHEL DESHAIES}

Michel Deshaies est professeur à l'Université de Lorraine. Il dirige le laboratoire de géographie LOTERR (Laboratoire d'observation des territoires) et ses recherches portent sur l'exploitation des ressources, les mines, les paysages et l'environnement. Michel.Deshaies@univ-lorraine.fr 